\title{
Normalized Transverse Emittance Reduction via Ionization Cooling in MICE 'Flip Mode'
}

\author{
Paul Bogdan Jurj* on behalf of the MICE collaboration ${ }^{1}$ \\ Department of Physics, Imperial College London, \\ London, United Kingdom \\ E-mail: paul.jurj13@imperial.ac.uk
}

Low emittance muon beams are central to the development of a Muon Collider and can significantly enhance the performance of a Neutrino Factory. The international Muon Ionization Cooling Experiment (MICE) has recorded several million individual muon tracks passing through a liquid hydrogen or a lithium hydride absorber and has demonstrated the ionization cooling of muon beams. A previous analysis used a restricted data set, and the beam matching was not perfect. In this analysis, beam sampling routines were employed to account for imperfections in beam matching at the entrance into the cooling channel and enable an improvement of the cooling measurement. A study of the normalized transverse emittance change in the MICE cooling channel set up in a flipped polarity magnetic field configuration is presented. Additionally, the evolution of the canonical angular momentum across the absorber is shown and the characteristics of the cooling effect are discussed.

\footnotetext{
*** The European Physical Society Conference on High Energy Physics (EPS-HEP2021), *** *** 26-30 July $2021 * * *$

*** Online conference, jointly organized by Universität Hamburg and the research center DESY ***
}

\footnotetext{
${ }^{1}$ The work described here was made possible by grants from Department of Energy and National Science Foundation (USA), the Istituto Nazionale di Fisica Nucleare (Italy), the Science and Technology Facilities Council (UK), the European Community under the European Commission Framework Programme 7 (AIDA project, grant agreement no. 262025, TIARA project, grant agreement no. 261905, and EuCARD), the Japan Society for the Promotion of Science and the Swiss National Science Foundation, in the framework of the SCOPES programme. We gratefully acknowledge all sources of support. We are grateful to the support given to us by the staff of the STFC Rutherford Appleton and Daresbury Laboratories and the Cockroft Institute. We acknowledge the use of Grid computing resources deployed and operated by GridPP in the UK, http://www.gridpp.ac.uk/.

${ }^{*}$ Speaker
} 


\section{The Muon Ionization Cooling Experiment}

Maximizing the beam intensity and maintaining a suitably small aperture in future facilities like a Neutrino Factory or a Muon Collider, require that the muon beam phase space volume be reduced (cooled) prior to acceleration. Ionization cooling, in which the muon beam is passed through a low- $Z$ absorber and subsequently accelerated, is the only viable technique to cool the beam. The MICE collaboration published the first demonstration of muon ionization cooling, in which the change in single particle amplitude distributions was studied [1].

In ionization cooling, the beam passes through an absorber material and loses both transverse and longitudinal momentum by ionizing atomic electrons. This reduces the phase space volume of the beam, decreasing the emittance. Simultaneously, multiple Coulomb scattering increases the angular divergence of the beam, generating emittance growth. The combined effect of the two processes is captured by the "cooling equation", which describes the rate of change of normalised transverse RMS emittance as follows,

$$
\frac{d \varepsilon_{\perp}}{d z} \approx-\frac{\varepsilon_{\perp}}{\beta^{2} E_{\mu}}\left|\frac{d E_{\mu}}{d z}\right|+\frac{\beta_{\perp}(13.6 \mathrm{MeV} / \mathrm{c})^{2}}{2 \beta^{3} E_{\mu} m_{\mu} X_{0}},
$$

where $\beta_{\perp}$ is the betatron function at the absorber, $\beta c$ and $E_{\mu}$ are the beam mean velocity and energy, $m_{\mu}$ is the muon mass, and $X_{0}$ is the radiation length of the absorber material. The cooling performance is increased by strongly focussing the beam at the absorber (low $\beta_{\perp}$ ) and using low-Z absorber materials, which have high $X_{0}\left|\frac{d E}{d z}\right|$.

In MICE, an upstream beamline [2] captures pions produced from proton interactions on a titanium target [3], allows the pions to decay into muons and transports the resulting muons into a cooling channel (CC). The cooling channel (Fig. 1 A) consists of 12 superconducting solenoid coils, symmetrically placed up and downstream of an absorber module which were configured depending on the beam momentum and required betatron function at the absorber. Particle identification detectors (PID) [4], placed up and downstream of the CC, are used to reject events in which pions enter the $\mathrm{CC}$ or electrons (decayed muons) exit the $\mathrm{CC}$. The scintillating fiber trackers [5, 6] measure the trajectories of the muons to determine their momenta before and after passing through the absorber. The absorbers used during data collection were a $65 \mathrm{~mm}$ lithium hydride disk $(\mathrm{LiH})$ and a 221 liquid hydrogen vessel ( $\mathrm{Full} \mathrm{LH}_{2}$ ), while an empty drift space (No absorber) and the empty vessel (Empty $\mathrm{LH}_{2}$ ) were used as controls. Data presented here were taken with the CC in a flipped polarity magnetic field configuration, to prevent the build-up of canonical angular momentum at the absorber. The solenoidal field strength evolution across the CC is shown in Fig. $1 \mathrm{~B}$.

\section{Emittance}

In this analysis, the effect of the absorber module on the 4-dimensional normalized transverse RMS emittance is studied. The beam emittance, a measure of the beam phase space volume, is calculated as follows

$$
\varepsilon_{\perp}=\frac{1}{m_{\mu} c} \sqrt[4]{|\Sigma|}
$$

where $\Sigma$ is the 4-dimensional covariance matrix and $m_{\mu}$ is the muon mass. 


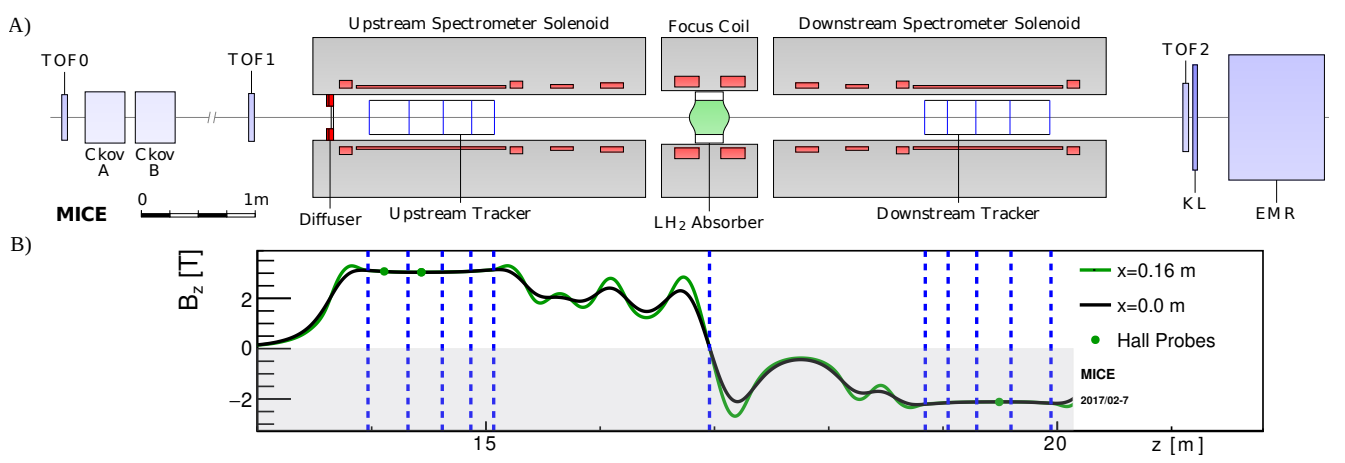

Figure 1: Schematic layout of the MICE cooling channel (A). Magnet coils are shown in red, the absorber in green and the various detetectors are individually marked. The modelled magnetic field along the length of the cooling channel is shown in (B) on-axis (black line) and at $160 \mathrm{~mm}$ from the beam axis (green line) in the horizontal plane. The measurements of Hall probes situated at $160 \mathrm{~mm}$ from the axis are also shown (green circles).

\section{Canonical Angular Momentum}

The canonical angular momentum of a particle, using a first order approximation of the magnetic vector potential, is given by

$$
L_{\text {canon }} \approx x p_{y}-y p_{x}+\frac{q r^{2} B_{z}}{2},
$$

where $x, y, p_{x}, p_{y}$ are the transverse phase space components, $r$ and $q$ are the radius and charge of the particle, respectively, and $B_{z}$ is the longitudinal magnetic field at the position of the particle.

\section{Analysis}

A set of cuts is applied to the individually reconstructed muon tracks to remove electron and pion impurities and select events with adequate reconstruction quality. The time of flight recorded upstream of the $\mathrm{CC}$ is required to be consistent with a muon with momentum as measured in the upstream tracker (135 - $145 \mathrm{MeV} / \mathrm{c}$ selection window) for each track. Each event is required to have a single, well-reconstructed track in both tracking detectors $\left(\chi^{2} / \mathrm{NDF}<8\right)$ and fully contained within the fiducial volume. All the events that are successfully reconstructed and pass all the cuts are combined to form the parent sample.

A beam selection procedure based on a rejection sampling algorithm is applied to the parent beam at the upstream reference plane (the tracker plane closest to the absorber), to select a beam with optics matched to the upstream tracker. As a result, the betatron function at the absorber is reduced, hence suppressing the heating effect due to multiple Coulomb scattering. Additionally, the selection procedure provides the flexibility of sampling beams with a range of input emittances.

\subsection{Emittance reduction}

Figure 2 shows the emittance change induced by the absorber module, for beams with input emittances in the $1.5-6.5 \mathrm{~mm}$ range. The beams are sampled from parent ensembles with nominal 

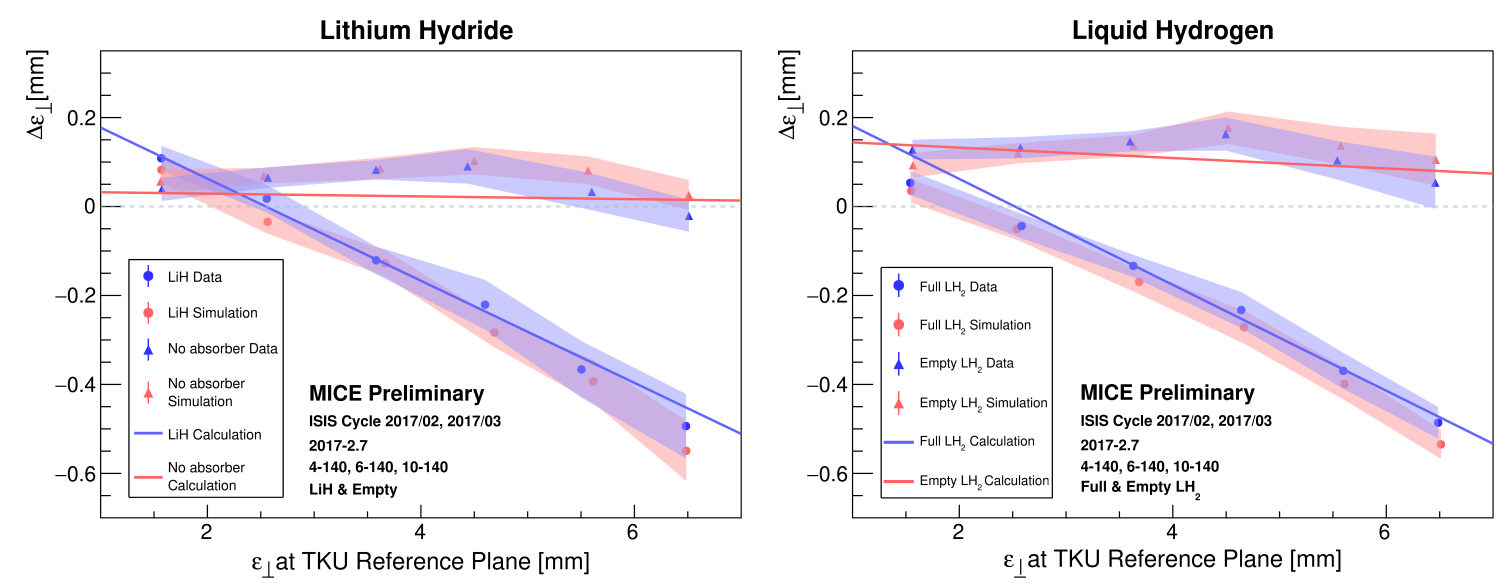

Figure 2: Emittance change between upstream and downstream tracker reference planes as function of beam emittance at the upstream tracker (TKU). Comparisons between (left) (circles) $\mathrm{LiH}$ and (triangles) No absorber data and (right) (circles) $\mathrm{Full} \mathrm{LH}_{2}$ and (triangles) Empty $\mathrm{LH}_{2}$ data indicate cooling in the presence of an ionizing material. The solid lines represent an approximate theoretical calculation for the (blue) absorber and (red) empty cases.

input emittance of 4, 6 and $10 \mathrm{~mm}$ and nominal input momentum of $140 \mathrm{MeV} / \mathrm{c}$. A correction is applied to both data and simulation, to account for detector resolution and transmission effects. The $\mathrm{LiH}$ and $\mathrm{Full} \mathrm{LH}_{2}$ absorbers demonstrate emittance reduction. This is a clear signal of ionization cooling, a direct consequence of the presence of an absorber material in the cooling channel. The control cases show no cooling effects - slight heating occurs due to optical aberrations in the empty channel (No absorber), while additional heating caused by the vessel windows is observed in the Empty $\mathrm{LH}_{2}$ case.

Good agreement between data, simulation and an approximate theoretical prediction is observed in all cases. The theoretical prediction is calculated from Eq. (1), with the beam parameters at the absorber and various aluminium windows informed from the truth simulation of the $3.5 \mathrm{~mm}$ beams. This is an approximation only, as it assumes the effective thickness of material traversed to be the same for all beams, while in reality this parameter increases with beam emittance (particularly for Empty $\mathrm{LH}_{2}$ and $\mathrm{Full} \mathrm{LH}_{2}$ ).

\subsection{Canonical angular momentum change}

The distributions of canonical angular momentum change between the upstream and downstream trackers are shown in Fig. 3. No net shift in the most probable value of the distributions occurs between the empty and absorber cases, as expected for a flipped field configuration. In contrast, data taken in a field that maintains polarity throughout the CC ('solenoid mode') show an increase in this quantity [7]. Similar behaviour is observed in data and simulation. The systematic effects are currently under study and are not accounted for here.

\section{Conclusion}

The preliminary MICE results in this work show a clear signal of ionization cooling in the presence of lithium hydride and liquid hydrogen absorbers. Ongoing efforts are focused on applying 


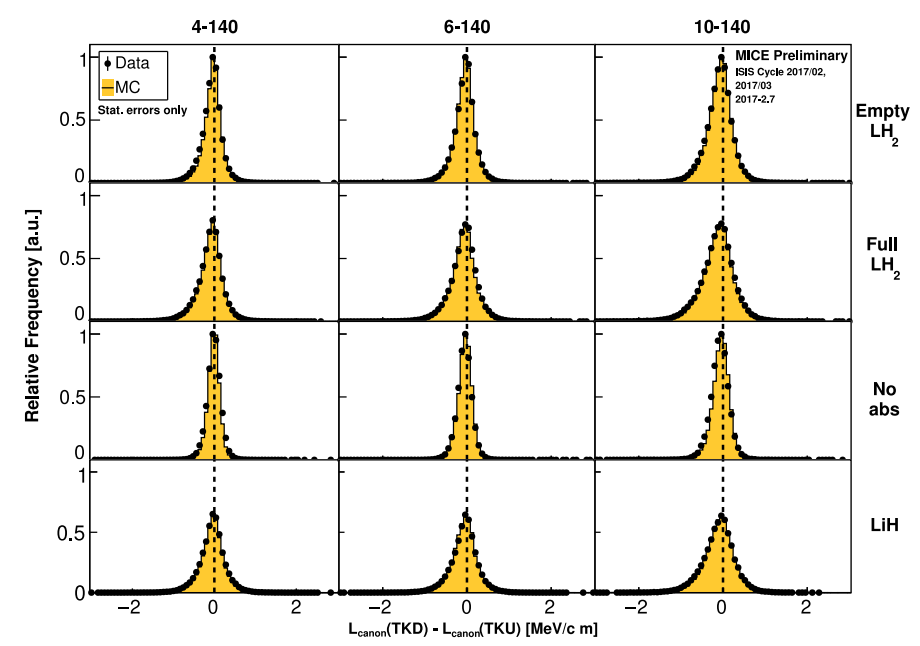

Figure 3: Comparison of (black circles) reconstructed data and (filled histogram) reconstructed simulation of canonical angular momentum change distributions, for the (left) 4, (middle) 6 and (right) $10 \mathrm{~mm}$ beams, in the four different CC settings.

the analysis to a variety of data sets, in order to study the cooling effect variation with input variables other than emittance, such as momentum and the betatron function. Furthermore, initial studies confirm canonical angular momentum conservation in a flipped field polarity configuration.

\section{References}

[1] MICE collaboration., M. Bogomilov, R. Tsenov, et al., "Demonstration of cooling by the Muon Ionization Cooling Experiment," Nature, vol. 578, pp. 53-59, Feb. 2020, https: //doi .org/10.1038/s41586-020-1958-9.

[2] M. Bogomilov et al., "The MICE Muon Beam on ISIS and the beam-line instrumentation of the Muon Ionization Cooling Experiment," JINST, vol. 7, pp. 5009, May 2012, http: //dx. doi.org/10.1088/1748-0221/7/05/P05009.

[3] C. N. Booth et al., "The design, construction and performance of the MICE target," JINST, vol. 8, pp. 3006 , Mar. 2013, http://dx . doi .org/10 . 1088/1748-0221/8/03/P03006.

[4] D. Orestano, “The detector system of the MICE experiment," Nucl. Inst. Phys. A, vol. 617, pp. 45-47, 2010.

[5] M. Ellis et al., "The design, construction and performance of the MICE scintillating fibre trackers," NIM A, vol. 659, pp. 136-153, Dec. 2011, http://dx.doi.org/10.1016/j . nima.2011.04.041.

[6] A. Dobbs et al., "The reconstruction software for the MICE scintillating fibre trackers," JINST, vol. 11, no. 12, pp. T12001, Dec. 2016,

[7] P. Kyberd, "Transverse Emittance Change and Canonical Angular Momentum Growth in MICE ‘Solenoid Mode’ with Muon Ionization Cooling,’ EPS-HEP2021, 2021. 